\title{
A case of Helicobacter pylori-negative early gastric adenocarcinoma with gastrointestinal phenotype
}

\section{다)(우우}

\author{
Authors \\ Masafumi Takatsuna ${ }^{1}$, Rie Azumi ${ }^{1}$, Takeshi Mizusawa', Hiroki Sato ${ }^{1}$, Ken-Ichi Mizuno', Takashi Kato ${ }^{2}$, Junji \\ Yokoyama', Yoichi Ajioka², Shuji Terai ${ }^{1}$
}

Institutions

1 Division of Gastroenterology and Hepatology, Graduate School of Medical and Dental Sciences, Niigata

University, Niigata, Japan

2 Division of Molecular and Diagnostic Pathology, Graduate School of Medical and Dental Sciences, Niigata University, Niigata, Japan

submitted 18.11.2020

accepted after revision 9.2.2020

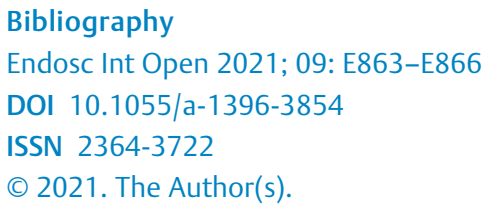
Commons Attribution-NonDerivative-NonCommercial License, permitting copying and reproduction so long as the original work is given appropriate credit. Contents may not be used for commercial purposes, or adapted, remixed, transformed or built upon. (https://creativecommons.org/licenses/by-nc-nd/4.0/)

Georg Thieme Verlag KG, Rüdigerstraße 14,

70469 Stuttgart, Germany

Corresponding author

Dr. Masafumi Takatsuna, Division of Gastroenterology and Hepatology, Graduate School of Medical and Dental Sciences, Niigata University, 1-757 Asahimachido-ri, Chuo-ku, Niigata,
Niigata 951-8510, Japan

Fax: +81-25-227-0776

masafumitakatsuna@gmail.com

\section{ABSTRACT}

A 40-year-old man with slightly depressed (0-IIc) type gastric cancer of the pyloric anterior gastric area underwent pre-operative screening for tetralogy of Fallot and endoscopic submucosal dissection (ESD) and was tested for Helicobacter pylori antigens and antibodies. Both tests were negative. He did not have a history of eradication. Pathological diagnosis of ESD showed a well-differentiated adenocarcinoma. The tumor was CD10-positive, MUC5AC-negative, and MUC6-confocal positive; it showed differentiation with gastrointestinal phenotype. Moreover, the tumor cells were lysozyme-positive, resembling Paneth cells. Mucosal glands exhibited intestinal metaplasia on the anal side of the tumor lesion. On the oral side of the tumor, metaplasia was non-existent, with normal pyloric glands present in the mucosal layer. The patient was not infected with H. pylori; however, intestinal metaplasia existed around the early gastric cancer. This suggested that the intestinal metaplasia occurred due to bile reflux, and the gastric neoplasia arose with the metaplasia without an $H$. pylori infection. This case may potentially help explain gastric cancer development in the absence of $H$. pylori infection.

\section{Introduction}

Helicobacter pylori infection is an important factor in the occurrence of gastric neoplasms; it induces atrophy and intestinal metaplasia of the gastric mucosa, and these changes increase the risk of gastric cancer [1]. Some reports have suggested that gastric cancer occurs in $\mathrm{H}$. pylori-negative patients, although most gastric cancers develop in $H$. pylori-infected patients [2]. We report a case of early gastric adenocarcinoma with a gastrointestinal phenotype in a patient without $H$. pylori infection.

\section{Case report}

A 40-year-old man with tetralogy of Fallot (TOF) tested negative for antibodies and antigens for $H$. pylori in the blood and stool, respectively. Moreover, he did not have a history of eradication. He underwent esophagogastroduodenoscopy because screening for TOF surgery revealed a gastric malignancy. The endoscopic view showed a moderately reddish, flat ridge with a depression (10-mm diameter) on the greater curvature of the pyloric antrum (>Fig.1a). Magnifying endoscopy with narrowband imaging revealed an irregular microsurface pattern; an irregular microvascular pattern with a demarcation line; presence of a white opaque substance in the depressed lesion 

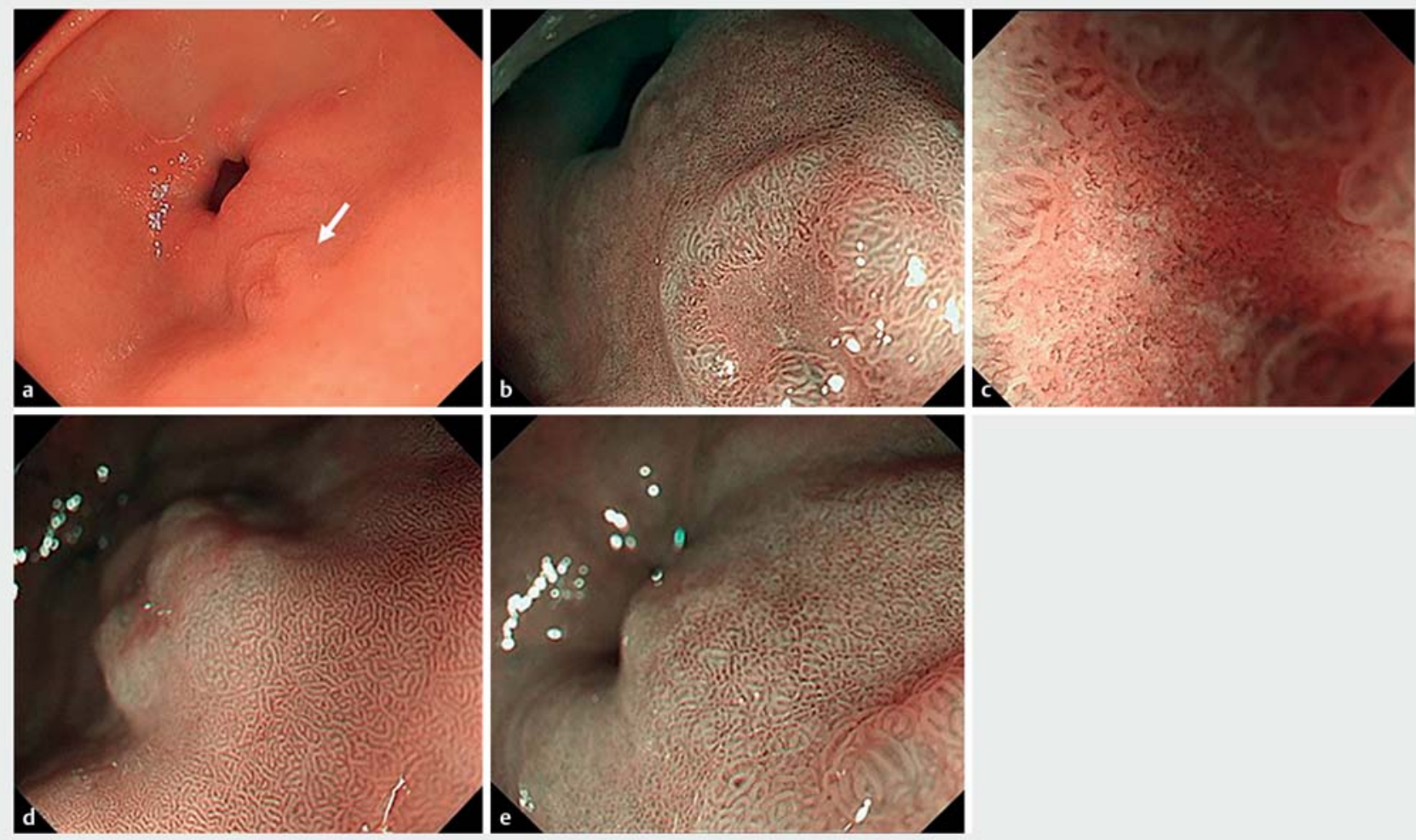

Fig. 1 a Depressed lesion on the greater curvature of the pyloric antrum under white light (white arrow), b lower magnified image of the depressed lesion, and $\mathbf{c}$ more highly magnified image using narrow-band imaging (NBI). $\mathbf{d}$ Magnified image of the mucosa of the oral side of the depressed lesion and $\mathbf{e}$ of the anal side of the depressed lesion.

( $\triangleright$ Fig.1b and $>$ Fig.1c); non-atrophic mucosa on the oral side surrounding the depressed lesion ( $>$ Fig.1d); and a fine ridge and villous structure, different from that on the oral side, on the anal side mucosa ( $\mathbf{F i g . 1 e ) . ~ B i o p s i e s ~ w e r e ~ p e r f o r m e d ~ a n d ~}$ the histopathological diagnosis was well-differentiated tubular adenocarcinoma. We diagnosed intra-mucosal gastric cancer and performed endoscopic submucosal dissection (ESD) 2 months later. Histopathological results revealed that the welldifferentiated tubular adenocarcinoma was confined to the mucosa of the depressed area ( $>$ Fig.2a). Some parts of the tumor cells possessed aerophilic fine granules ( $\mathbf{F i g . 2 b}$ ) and were positive for lysozyme on immunohistochemical and phosphotungstic acid-hematoxylin staining ( $>$ Fig.2c and $>$ Fig.2d). Immunohistochemical staining showed that the tumor cells were positive for CD10 (\$Fig.2e) and MUC2 (> Fig.2f), negative for MUC5AC ( $\mathbf{F i g . 2} \mathbf{g}$ ), and positive for MUC6 ( $\mathbf{F i g . 2} \mathbf{h}$ ). These results suggested that the tumor cells had characteristics of the gastrointestinal phenotype. On the anal side of the tumor, in the mucosal layer of the non-tumorous region, the metaplasia contained intestinal-like cells with characteristics of goblet and Paneth cells (> Fig.3a). Immunohistochemical staining showed that the mucosa cells were positive for CD10 ( $>$ Fig.3b). In contrast, on the oral side of the tumor, there was no metaplasia and the mucosal layer had normal pyloric glands ( Fig.3c).

\section{Discussion}

We diagnosed that this patient was not infected with $H$. pylori, which was further supported by negative results for the antibody and antigen tests for $\mathrm{H}$. pylori and the absence of a history of eradication. However, we did not evaluate the patient according to the Updated Sydney System for the classification and grading of gastritis. This assessment may offer further evidence for $H$. pylori-negativity. Moreover, a history of $H$. pylori infection could not be excluded because of the intestinal metaplasia in this case. The diagnosis in the present case was $H$. pylori-negative, intramucosal, well-differentiated adenocarcinoma with gastrointestinal phenotype. However, the neoplastic tissue was diagnosed as intestinal-type adenoma with highgrade atypia according to the World Health Organization classification [3]. Kato et al. reported that many cases of $\mathrm{H}$. pylorinegative gastric cancer exhibit the gastrointestinal phenotype [4]. Paneth cells are characterized by acidophilic granules in the cytoplasm and are normally found in the small intestine region [5, 6]. However, Paneth-like cells are found in the stomach in intestinal metaplasia and adenomas [6,7]. We concluded that the tumor cells in the present case had the gastrointestinal phenotype and the characteristic differentiation of Paneth cells.

Kotani et al. and Ozaki et al. reported that an H. pylori-negative intramucosal, well-differentiated, gastric adenocarcinoma 


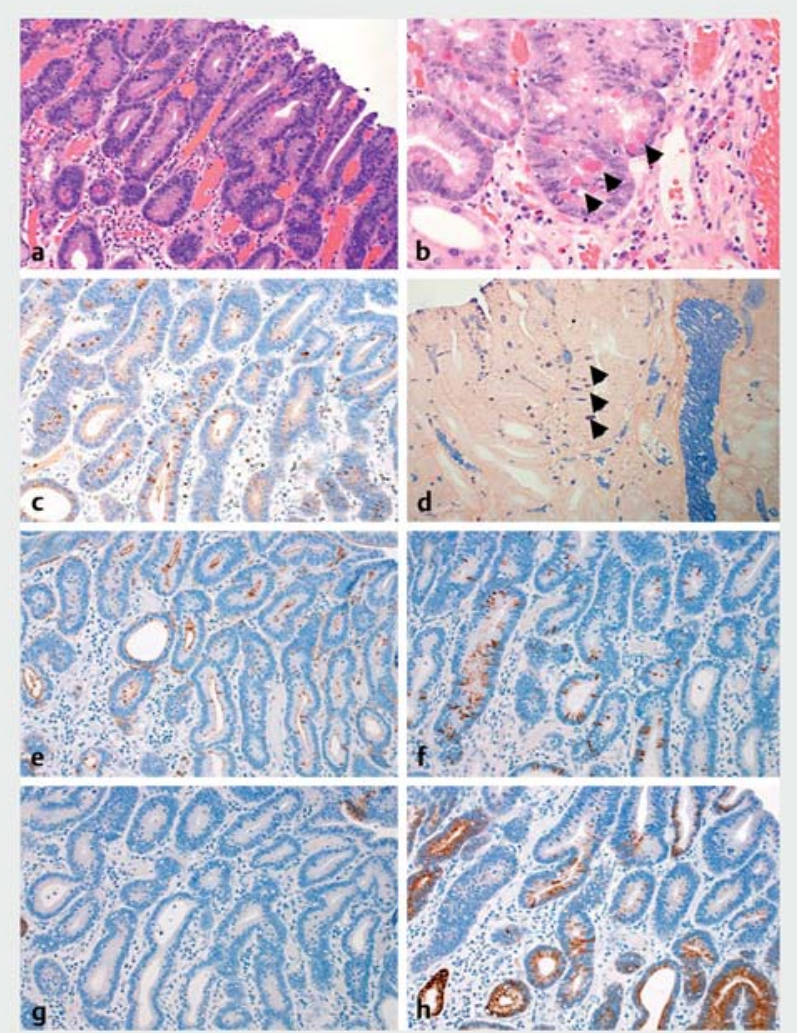

- Fig. 2 a Histopathological image of the endoscopic submucosal dissection. The region of the well-differentiated tubular adenocarcinoma exists in the depressed lesion $(\times 200)$. b The cancer cells have aerophilic fine granules (black arrow) $(\times 400)$ and are positive for lysozyme on $\mathrm{c}$ immunohistochemical staining $(\times 200)$ and $\mathbf{d}$ phosphotungstic acid hematoxylin (PTAH) staining (black arrow) ( $\times 400)$. e Immunohistochemical staining shows the tumor cells are e positive for CD10 $(\times 200), \mathbf{f}$ positive for MUC2 (confocal, $\times 200)$, g negative for MUC5AC $(\times 200)$, and $\mathbf{h}$ positive for MUC6 (confocal, $\times 200$ ). with intestinal phenotype in the antral mucosa was a 0-Ilc type lesion, and that neither atrophy nor intestinal metaplasia was present around the lesion $[8,9]$. These two reports did not mention whether the tumor cells were positive for lysozyme. Our case suggests that lysozyme-positive tumor cells arise from intestinal metaplasia of the gastric mucosa. Moreover, we suggest that in $H$. pylori-negative cases, the development of gastrointestinal phenotype adenocarcinoma occurs through a different mechanism than that of intestinal type adenocarcinoma, even though the morphological features of the tumors are similar.

Correa et al. proposed a series of processes as a hypothesis for gastric cancer development: normal mucosa $\rightarrow$ superficial gastritis $\rightarrow$ atrophic gastritis $\rightarrow$ intestinal metaplasia $\rightarrow$ dysplasia $\rightarrow$ gastric cancer [10]. H. pylori infection of the normal gastric mucosa causes superficial gastritis, which then progresses to atrophic gastritis. It is thought that the atrophic mucosa develops into intestinal metaplasia due to the involvement of environmental and host genetic factors; hence, differentiated gastric cancer may develop from intestinal metaplasia $[11,12]$. In patients who are $H$. pylori-negative, many factors, such as embryological factors, genetic factors, and the molecular biology of intestinal metaplasia, can affect the gastric mucosa. Other reports propose that there is a relationship between bile acid reflux in the stomach and the risk of atrophic gastritis and intestinal metaplasia $[13,14]$. Therefore, in this patient, who was $H$. pylori-negative, it was hypothesized that intestinal metaplasia occurred in the pyloric region due to bile regurgitation and gastric tumor development. However, the mechanism of carcinogenesis in this case is only hypothetical and may not be the actual mechanism underlying uninfected gastric cancer because a history of $H$. pylori infection could not be excluded.

\section{Conclusions}

The present case is of interest as it can help expand understanding of gastric carcinogenesis in patients who are not infected with $H$. pylori.

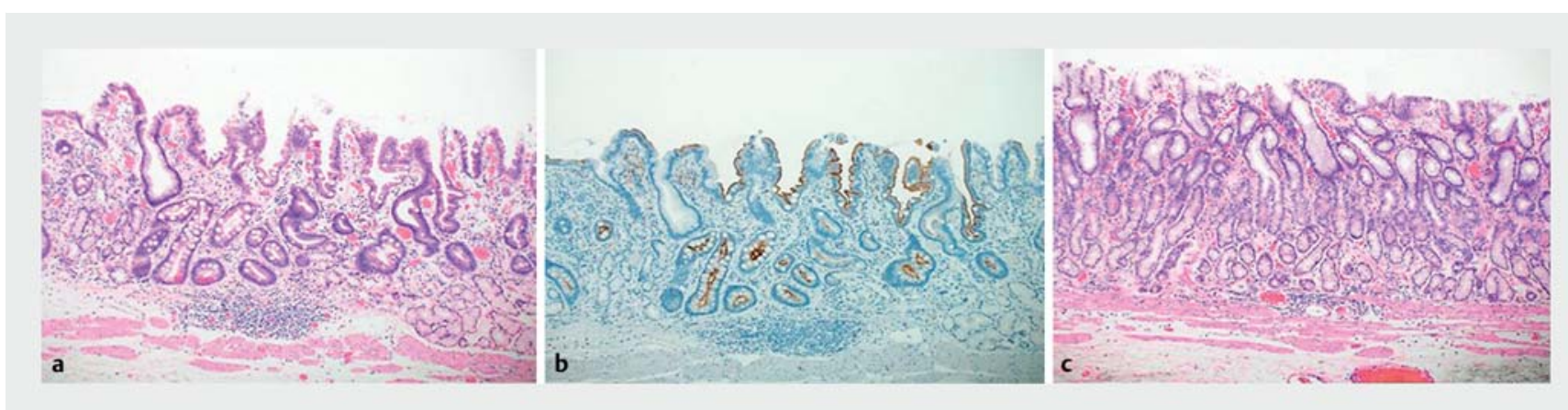

- Fig. 3 a Non-tumorous mucosal layer with intestinal metaplasia displaying characteristics of goblet cells and similar to Paneth cells $(\times 100)$. b Immunohistochemical staining shows that anal-side mucosal cells are positive for CD10 ( $\times 100)$. c On the oral side of the tumor, metaplasia is non-existent, and normal pyloric glands are present in the mucosal layer $(\times 100)$. 


\section{Competing interests}

The authors declare that they have no conflict of interest.

\section{References}

[1] Matsushita T, Yamada N, Kato S et al. Helicobacter pylori infection, mucosal atrophy and intestinal metaplasia in Asian populations: a comparative study in age-, gender-, and endoscopic diagnosis-matched subjects. Helicobacter 2003; 8: 29-35

[2] Uemura N, Okamoto S, Yamamoto $S$ et al. Helicobacter pylori nfection and the development of gastric cancer. N Engl J Med 2001; 345: 784-789

[3] Nagtegaal ID, Odze RD, Klimstra D. WHO classification of Tumors of the Digestive System. 5th ed. Lyon: IARC. 2019

[4] Kato S, Matsukura N, Tsukada K et al. Helicobacter pylori infectionnegative gastric cancer in Japanese hospital patients: incidence and pathological characteristics. Cancer Sci 2007; 98: 790-794

[5] Bevins CL. The Paneth cell and the innate immune response. Curr Opin Gastroenterol 2004; 20: 572-580

[6] Wong WM, Stamp GW, Elia G et al. Proliferative populations in intestinal metaplasia: evidence of deregulation in Paneth and goblet cells, but not endocrine cells. J Pathol 2000; 190: 107-113
[7] Fukasawa T, Suzuki S, Fujii H. A case report of early gastric cancer with Paneth-like tumor cells. Nihon Shokakibyo Gakkai Zasshi 2006; 103: 1251-1256

[8] Kotani S, Miyaoka Y, Fujiwara A et al. Intestinal-type gastric adenocarcinoma without Helicobacter pylori infection successfully treated with endoscopic submucosal dissection. Clin J Gastroenterol 2016; 9: 228-32

[9] Ozaki Y, Suto H, Nosaka T et al. A case of Helicobacter pylori-negative intramucosal well-differentiated gastric adenocarcinoma with intestinal phenotype. Clin J Gastroenterol 2015; 8: 18-21

[10] Correa P, Cuello C, Duque E. Carcinoma and intestinal metaplasia of the stomach in Colombian migrants. J Natl Cancer Inst 1970; 44: 297 306

[11] Uemura N, Okamoto S, Yamamoto $S$ et al. Helicobacter pylori infection and the development of gastric cancer. N Engl J Med 2001; 345: 784-789

[12] Asaka M, Kato M, Kudo M et al. Relationship between Helicobacter pylori infection, atrophic gastritis and gastric carcinoma in a Japanese population. Eur J Gastroenterol Hepatol 1995; 7: S7-S10

[13] Karita M. Relationship of bile acid level in the gastric juice to intestinal metaplasia in the stomach (II). Gastrointest Endosc 1987; 29: 16711682

[14] Tatsugami M, Ito M, Tanaka S et al. Bile acid promotes intestinal metaplasia and gastric carcinogenesis. Cancer Epidemiol Biomarkers Prev 2012; 21: 2101-2107 